\title{
New phenyl derivatives from endophytic fungus Botryosphaeria sp. SCSIO KcF6 derived of mangrove plant Kandelia candel
}

Zhi-ran Ju ${ }^{\mathrm{a}, \mathrm{b}, 1}$, Xiaochu Qin ${ }^{\mathrm{c}, 1}$, Xiu-ping Lin ${ }^{\mathrm{b}}$, Jun-feng Wang ${ }^{\mathrm{b}}$, Kumaravel

Kaliyaperumal $^{\mathrm{b}}$, Yong-qi Tian ${ }^{\mathrm{b}}$, Juan Liu ${ }^{\mathrm{b}}$, Fen Liu ${ }^{\mathrm{a}}$, Zhengchao Tu${ }^{\mathrm{c},}$, Shi-hai Xu ${ }^{\mathrm{a},}{ }^{*}$, Yonghong Liu ${ }^{\text {b,* }}$

${ }^{a}$ Department of Chemistry, Jinan University, Guangzhou 510632, China;

${ }^{\mathrm{b}}$ CAS Key Laboratory of Tropical Marine Bio-resources and Ecology/Guangdong Key Laboratory of Marine Materia Medica/ RNAM Center for Marine Microbiology, South China Sea Institute of Oceanology, Chinese Academy of Sciences, Guangzhou 510301, China;

${ }^{\mathrm{c}}$ Laboratory of Molecular Engineering and Laboratory of Natural Product Synthesis, Guangzhou Institutes of Biomedicine and Health, Chinese Academy of Sciences, Guangzhou 510530, China

${ }^{1}$ The first two authors contributed equally to this work.

*Corresponding author

*Correspondence to Y. Liu, CAS Key Laboratory of Tropical Marine Bio-resources and Ecology/Guangdong Key Laboratory of Marine Materia Medica/ RNAM Center for Marine Microbiology, South China Sea Institute of Oceanology, Chinese Academy of Sciences, Guangzhou 510301, China. Tel./Fax: +86-020-8902-3244.

E-mail address: yonghongliu@scsio.ac.cn (Y. Liu).

Dr. Shihai Xu

Department of Chemistry, Jinan University, Guangzhou 510632, China

E-mail: txush@jnu.edu.cn

Dr. Zhengchao Tu, Guangzhou Institutes of Biomedicine and Health, Chinese Academy of Sciences, Guangzhou 510530, China, E-mail: tu_zhengchao@gibh.ac.cn 


\section{ABSTRACT}

Two new phenyl derivatives ( 1 and $\mathbf{3}$ ), along with two new natural products (4 and 5), and three known compounds (2, 6, and 7), were isolated from an endophytic fungus Botryosphaeria sp. SCSIO KcF6. The structures of these compounds 1-7 were elucidated by the extensive 1D and 2D-NMR and HRESIMS Data analysis, and compared with those of reported data. The absolute configuration of the compounds $\mathbf{1}$ and 3 were assigned by optical rotation and $\mathrm{CD}$ data. The isolated compounds were evaluated for their cytotoxic, anti-inflammatory (COX-2), and antimicrobial activities. Compound 3 exhibited a specific COX-2 inhibitory activity with the $\mathrm{IC}_{50}$ value of $1.12 \mu \mathrm{M}$.

Keywords: Endophytic fungus, Botryosphaeria sp. SCSIO KcF6, COX-2 inhibitory activity 


\section{Contents}

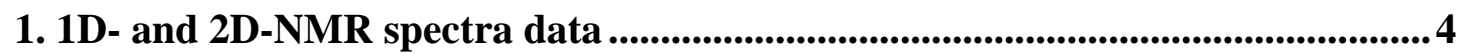

1.1 Fig. 1 The ${ }^{1}$ H-NMR spectrum of compound 1 .............................................. 4

1.2 Fig. 2 The ${ }^{13}$ C-NMR (DEPT) spectrum of compound 1 .................................5 5

1.3 Fig. 3 The HSQC spectrum of compound 1 .............................................6

1.4 Fig. 4 The HMBC spectrum of compound 1 ............................................... 7

1.5 Fig. 5 The ${ }^{1} \mathrm{H}^{1}{ }^{1} \mathrm{H}$ COSY spectrum of compound 1 ...........................................8

1.6 Fig. 6 The ${ }^{1}$ H-NMR spectrum of compound 3 .................................................9

1.7 Fig. 7 The ${ }^{13}$ C-NMR (DEPT) spectrum of compound 3 ................................ 10

1.8 Fig. 8 The HSQC spectrum of compound 3............................................11

1.9 Fig. 9 The HMBC spectrum of compound 3 .................................................12

1.10 Fig. 10 The ${ }^{1} \mathrm{H}-{ }^{1} \mathrm{H}$ COSY spectrum of compound 3 ................................... 13

2. The chemical structure of compounds 1-7..................................................... 14

2.1 Fig. 11 The chemical structure of compounds 1-7 ................................... 14

2.2 Fig. 12 HMBC correlations of compound 1 ...................................................... 15

2.3 Fig. 13 HMBC correlations of compound 3 ................................................... 15

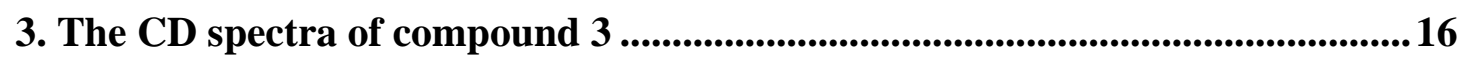

3.1 Fig. 14 The CD spectra of compound 3 ....................................................16

4. Spectroscopic data of the isolated compounds ................................................17

4.1 Compound 2 ................................................................................................17

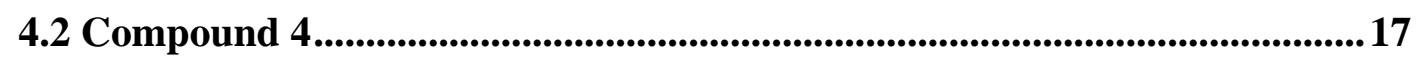

4.3 Compound 5 .............................................................................................................. 17

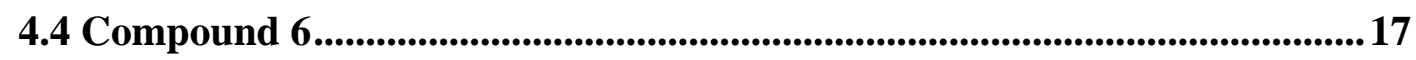

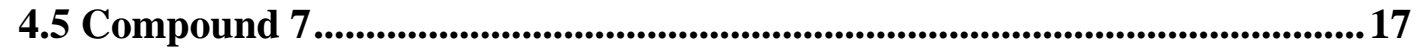

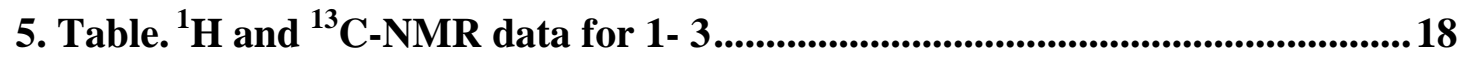

5.3 Table $1 .{ }^{1} \mathrm{H}$ and ${ }^{13} \mathrm{C}-\mathrm{NMR}$ data for 1 and 2 ............................................... 18

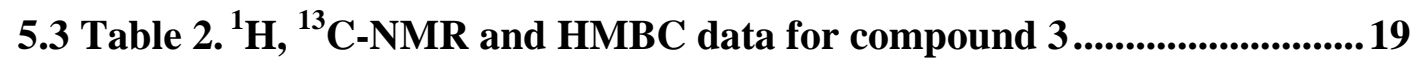

6. COX-2 inhibitory value of Compound 3................................................................ 20

6.1. Graphics of COX-2 inhibitory value of Compound 3...............................20 
1. 1D- and 2D-NMR spectra data

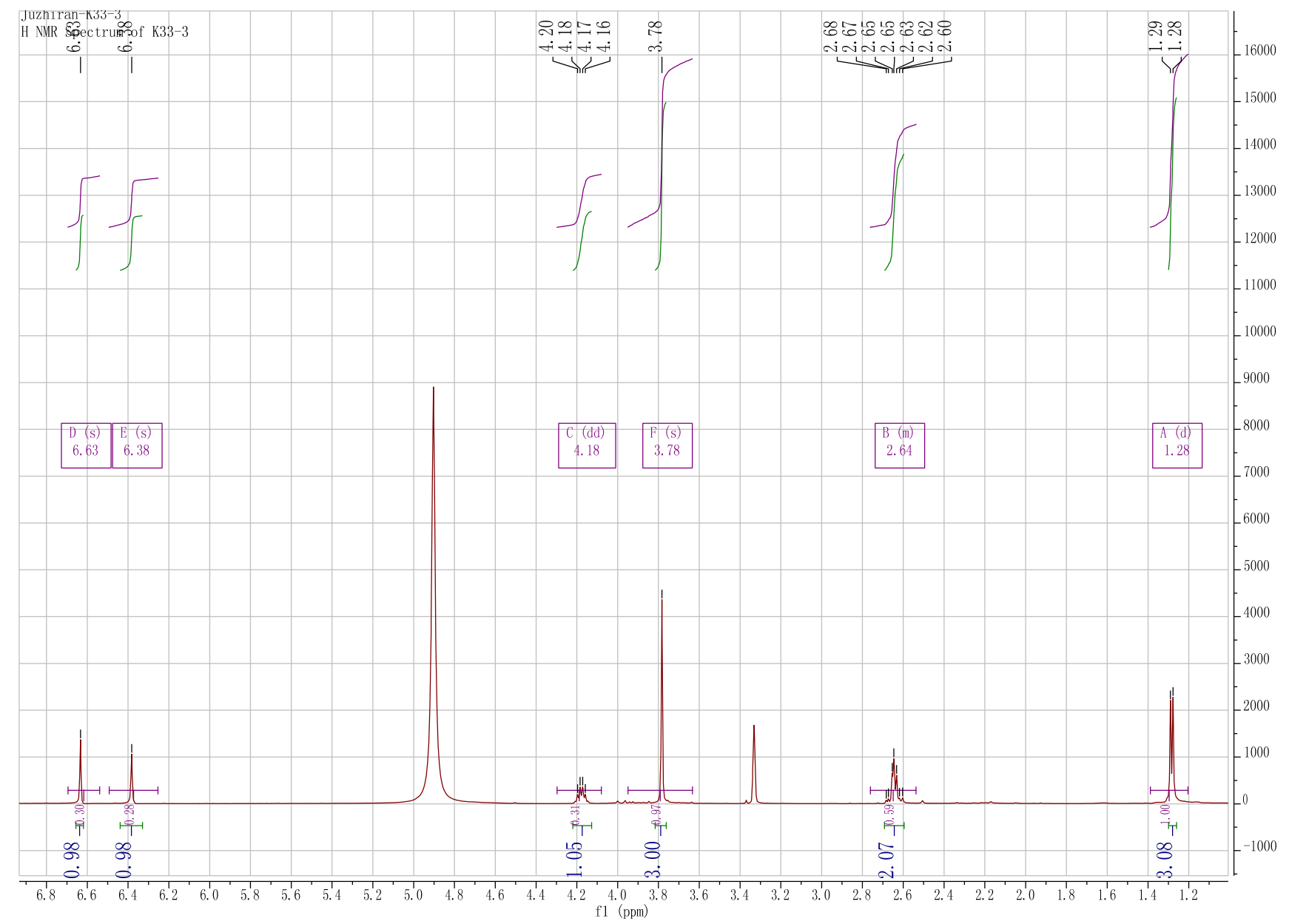

Fig. 1 The ${ }^{1} \mathrm{H}-\mathrm{NMR}$ spectrum of compound $\mathbf{1}(500 \mathrm{MHz}$ in MeOD) 


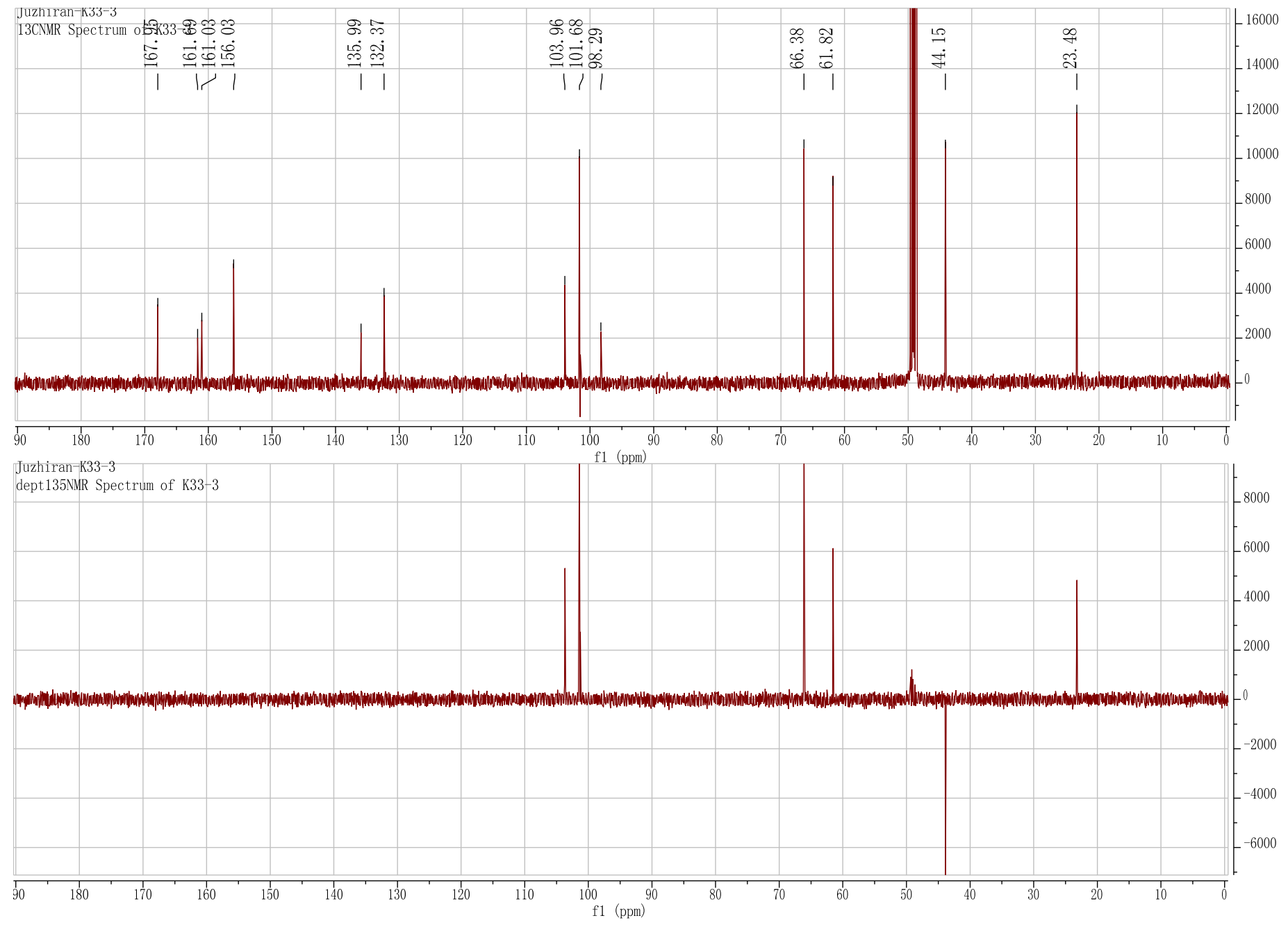

Fig. 2 The ${ }^{13} \mathrm{C}-\mathrm{NMR}$ (DEPT) spectrum of compound $\mathbf{1}(125 \mathrm{MHz}$ in MeOD) 


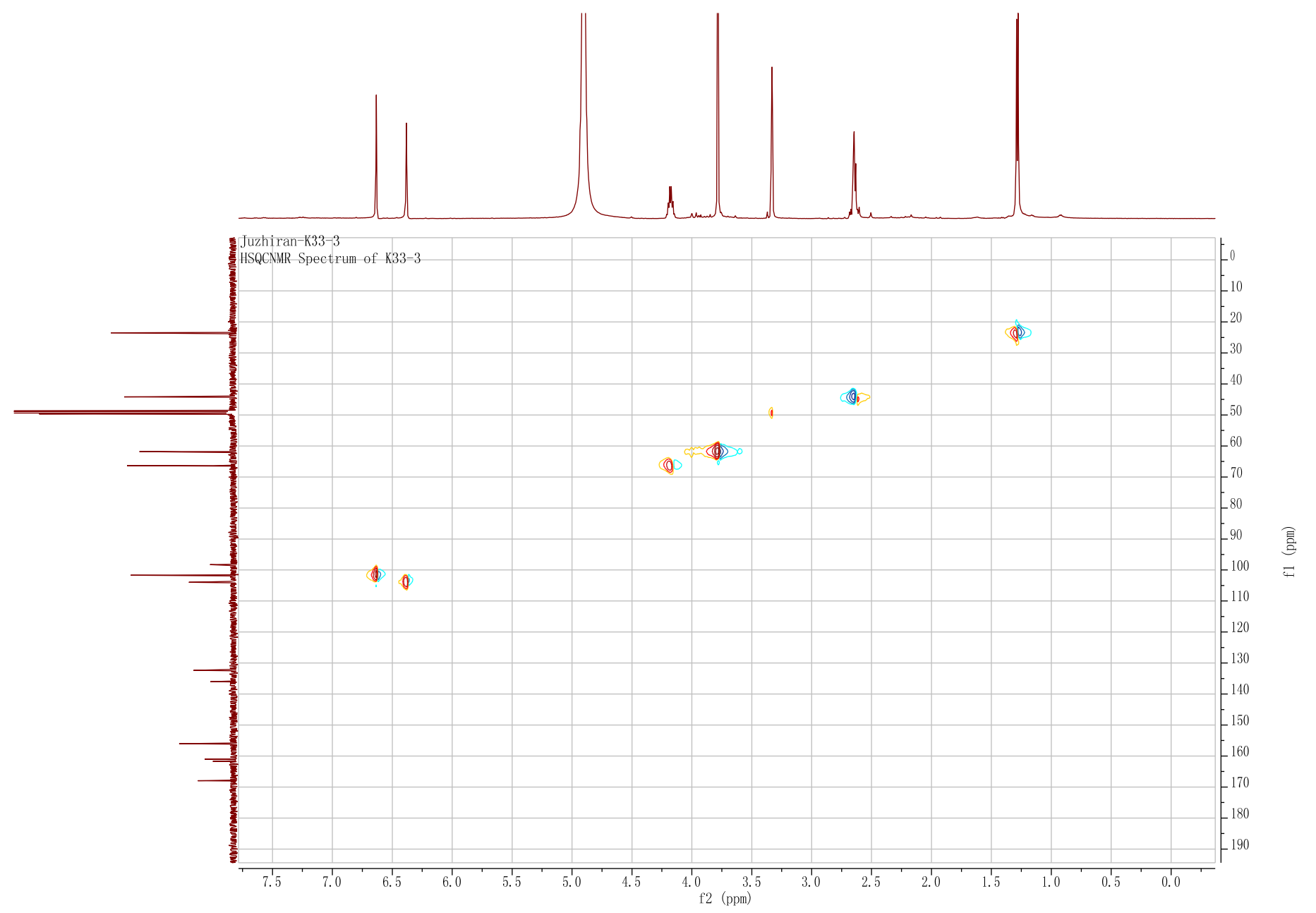

Fig. 3 The HSQC spectrum of compound 1 


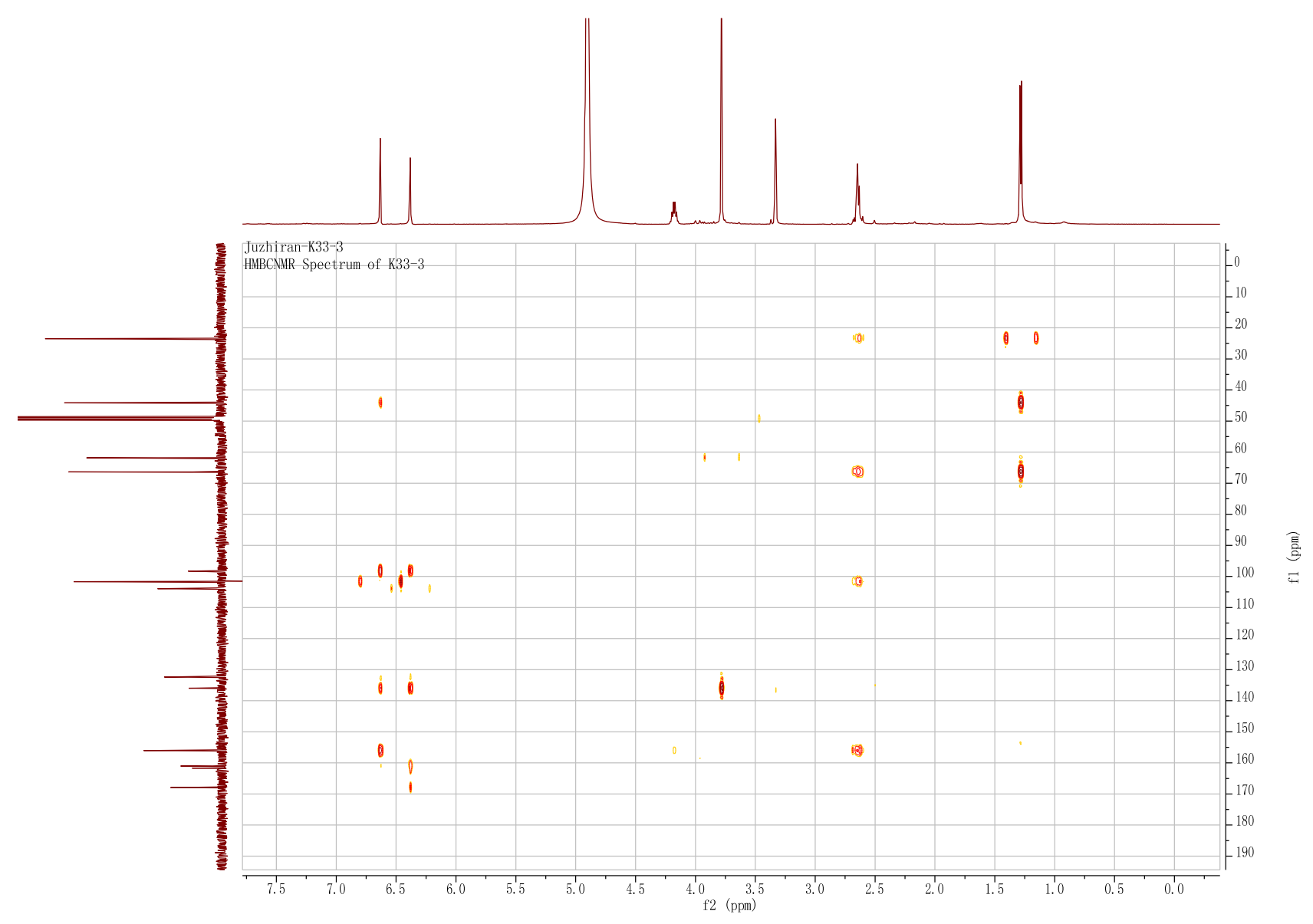

Fig. 4 The HMBC spectrum of compound 1 


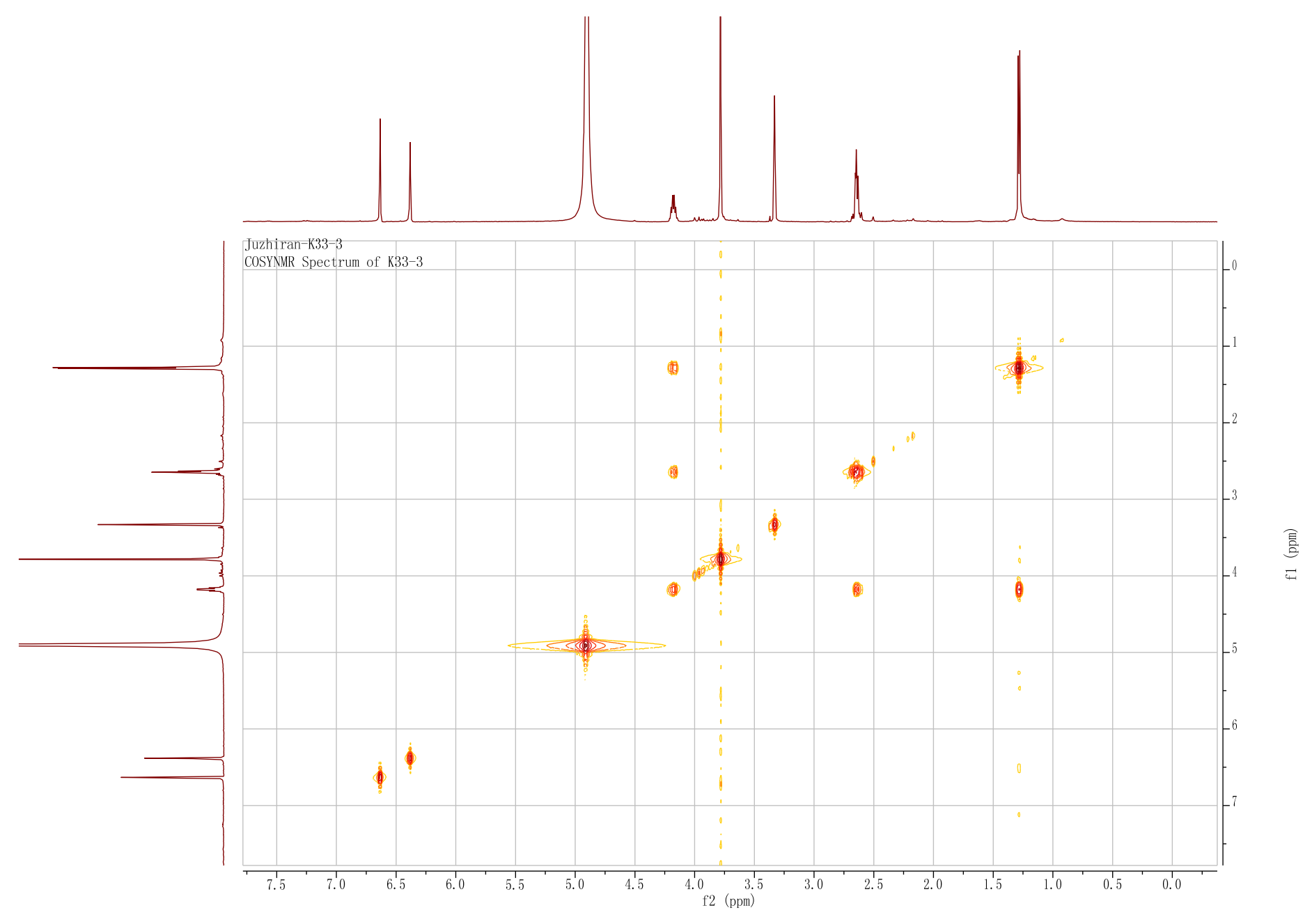

Fig. 5 The ${ }^{1} \mathrm{H}-{ }^{1} \mathrm{H}$ COSY spectrum of compound 1 


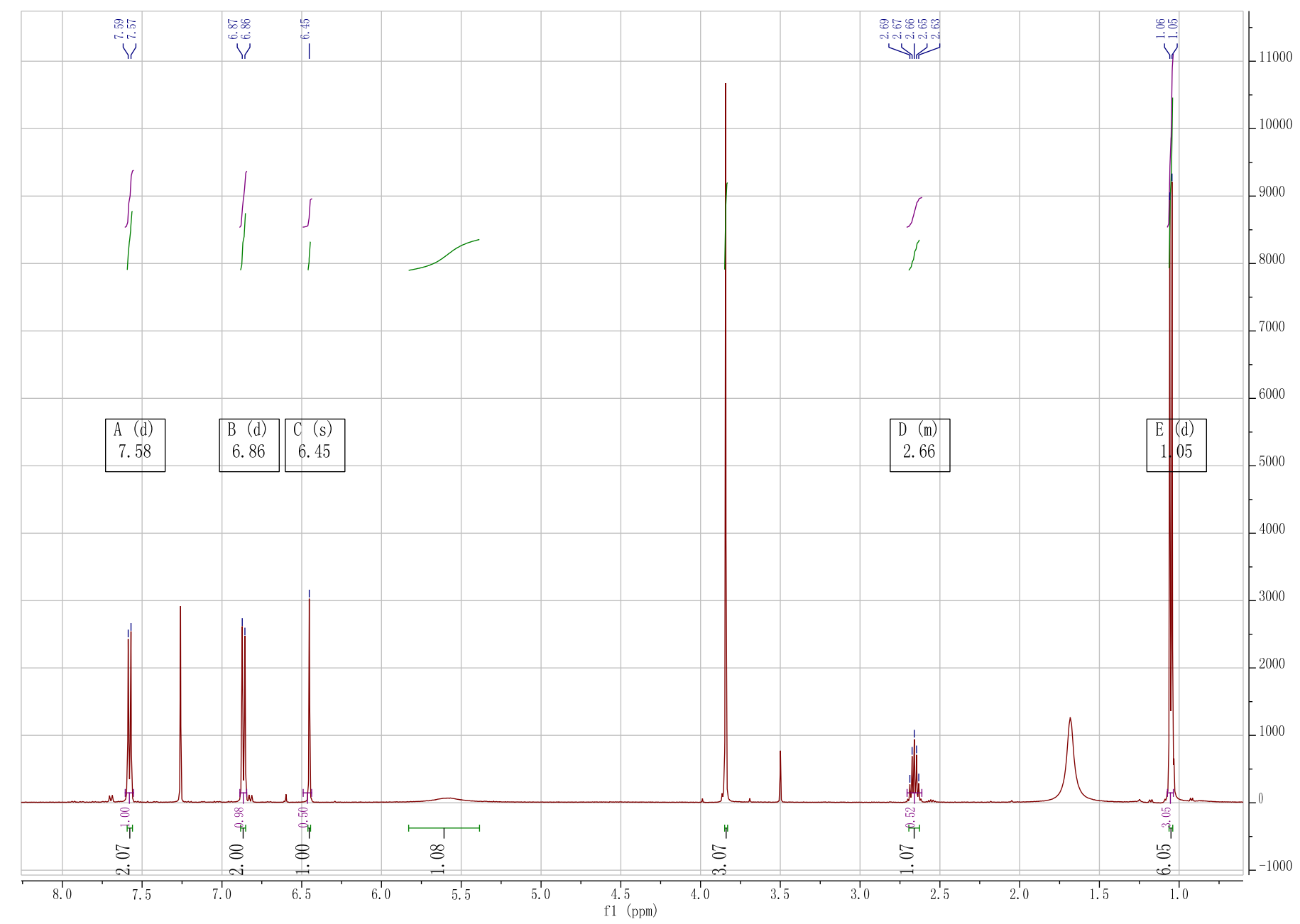

Fig. 6 The ${ }^{1} \mathrm{H}-\mathrm{NMR}$ spectrum of compound $3\left(500 \mathrm{MHz}\right.$ in $\left.\mathrm{CDCl}_{3}\right)$ 

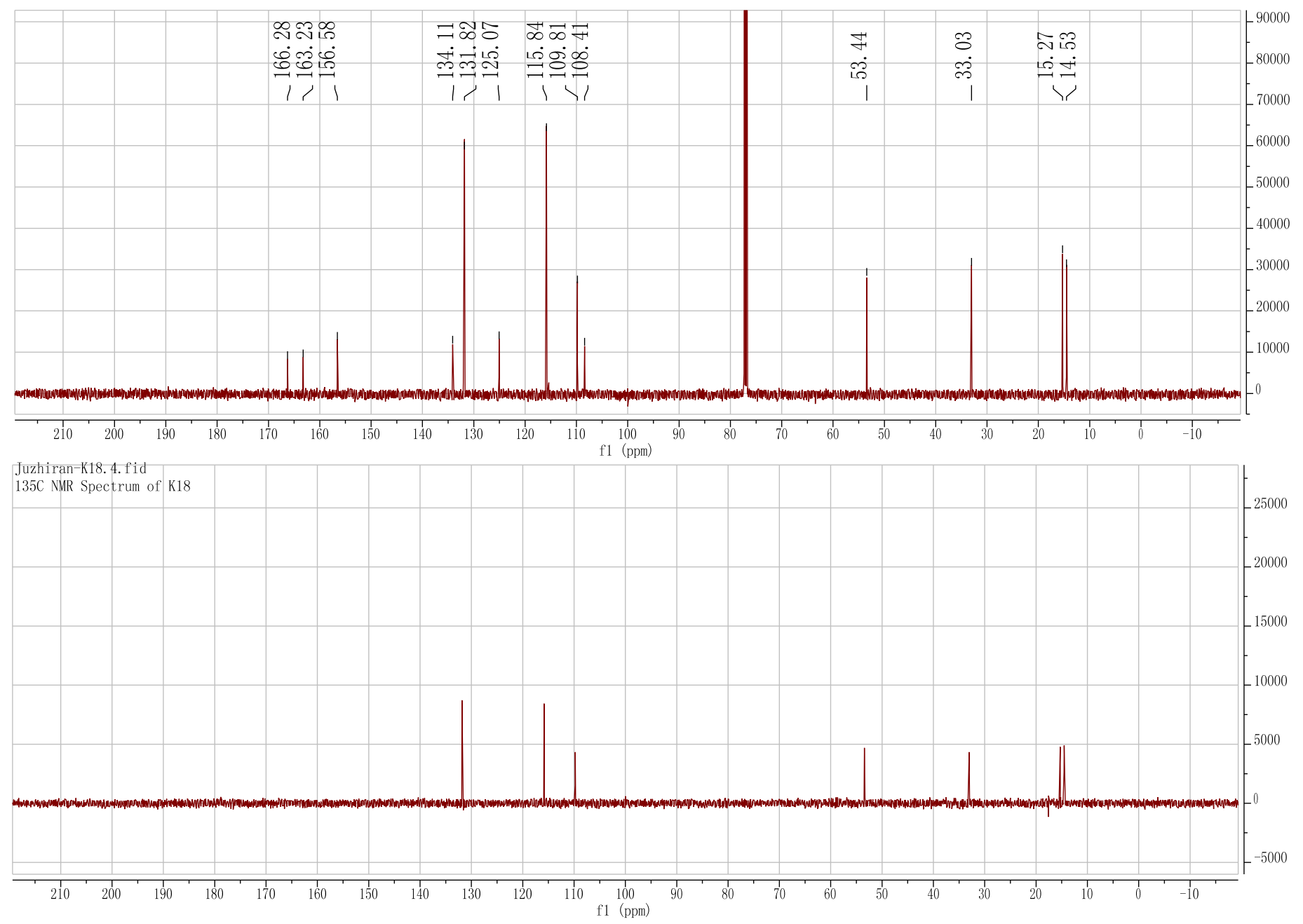

Fig. 7 The ${ }^{13} \mathrm{C}-\mathrm{NMR}$ (DEPT) spectrum of compound $3\left(125 \mathrm{MHz}\right.$ in $\left.\mathrm{CDCl}_{3}\right)$ 


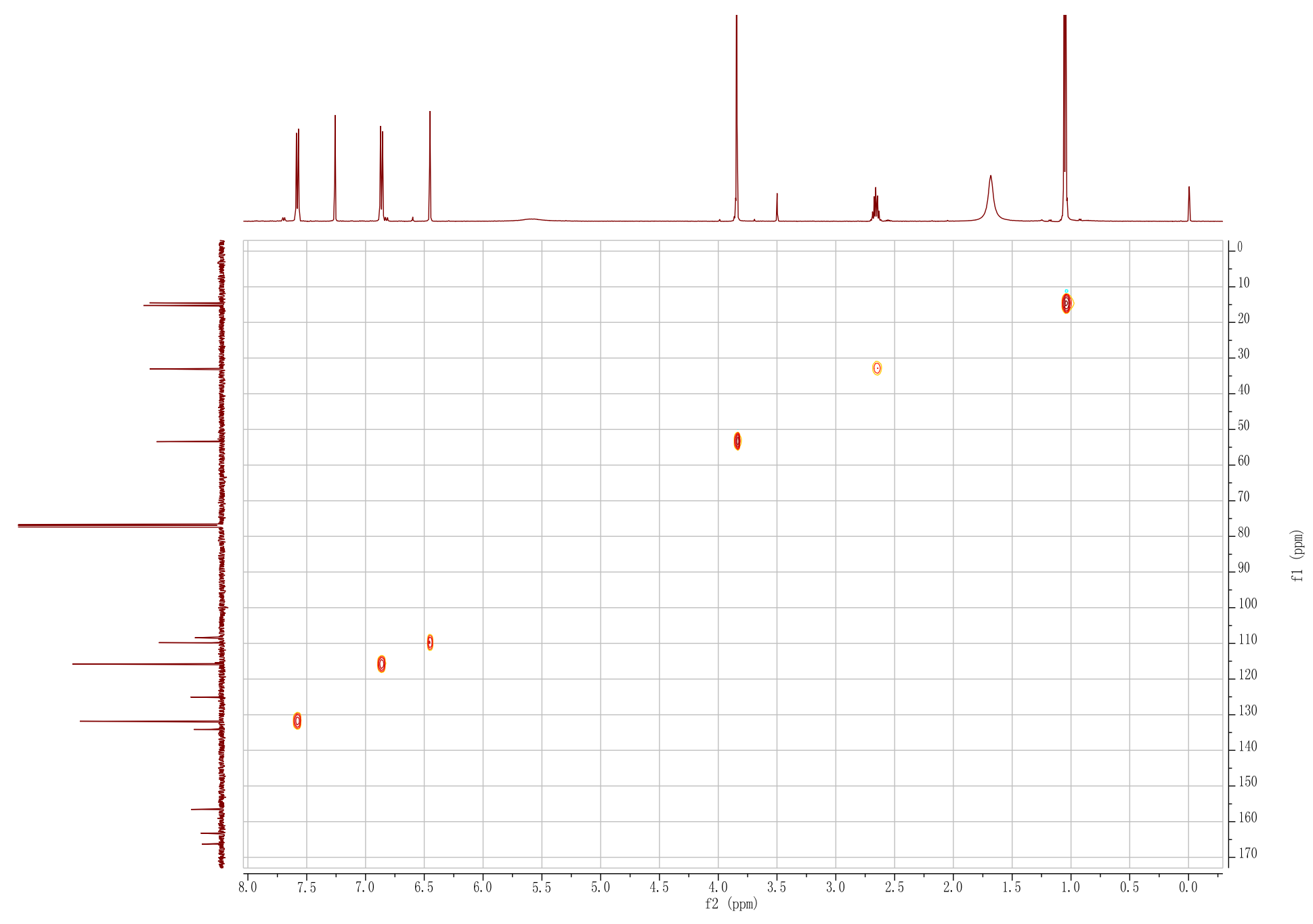

Fig. 8 The HSQC spectrum of compound 3 


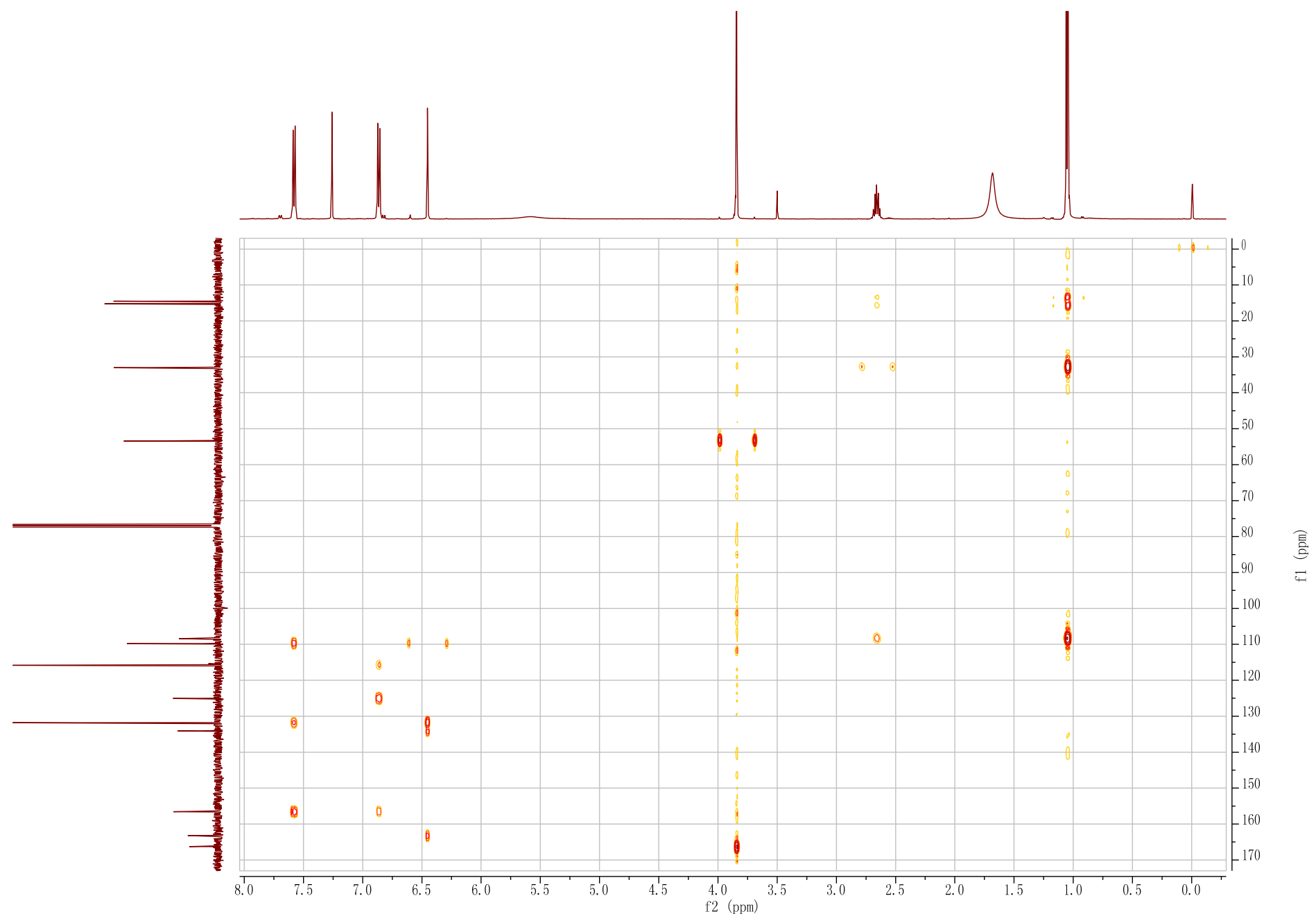

Fig. 9 The HMBC spectrum of compound 3 


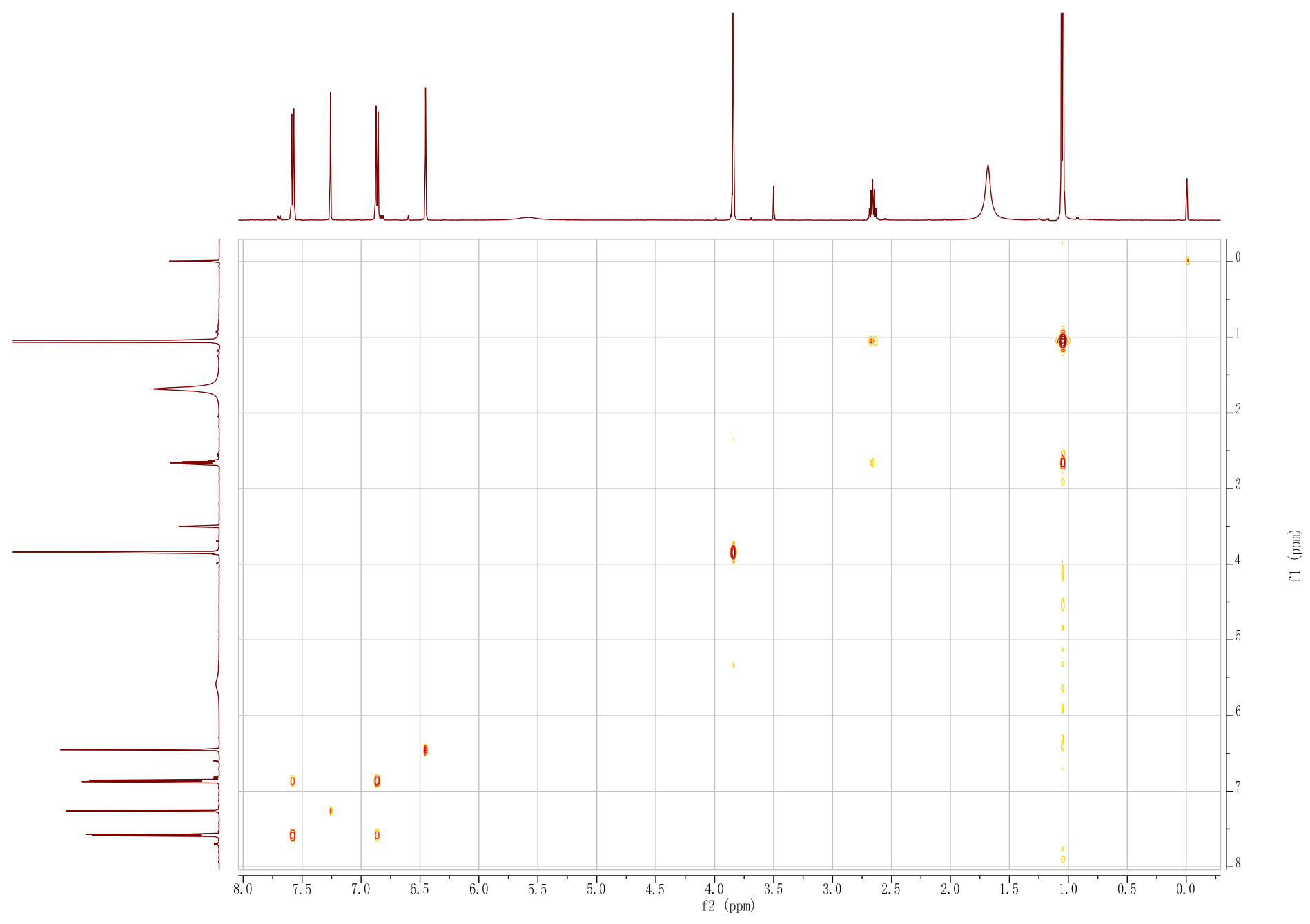

Fig. 10 The ${ }^{1} \mathrm{H}-{ }^{1} \mathrm{H}$ COSY spectrum of compound 3 
2. The chemical structure of compounds 1-7

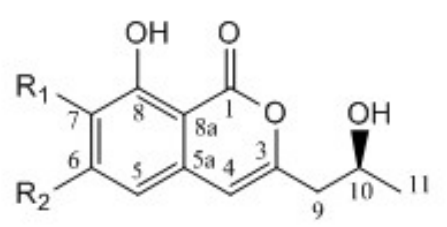

I: $\mathrm{R}_{1}=\mathrm{OH}, \mathrm{R}_{2}=\mathrm{OCH}_{3}$ 2: $\mathrm{R}_{1}=\mathrm{H}, \mathrm{R}_{2}=\mathrm{OH}$

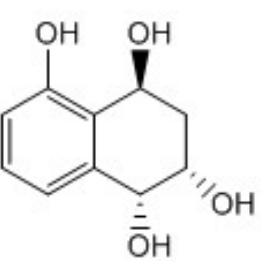

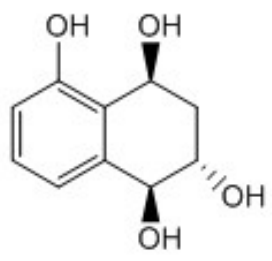
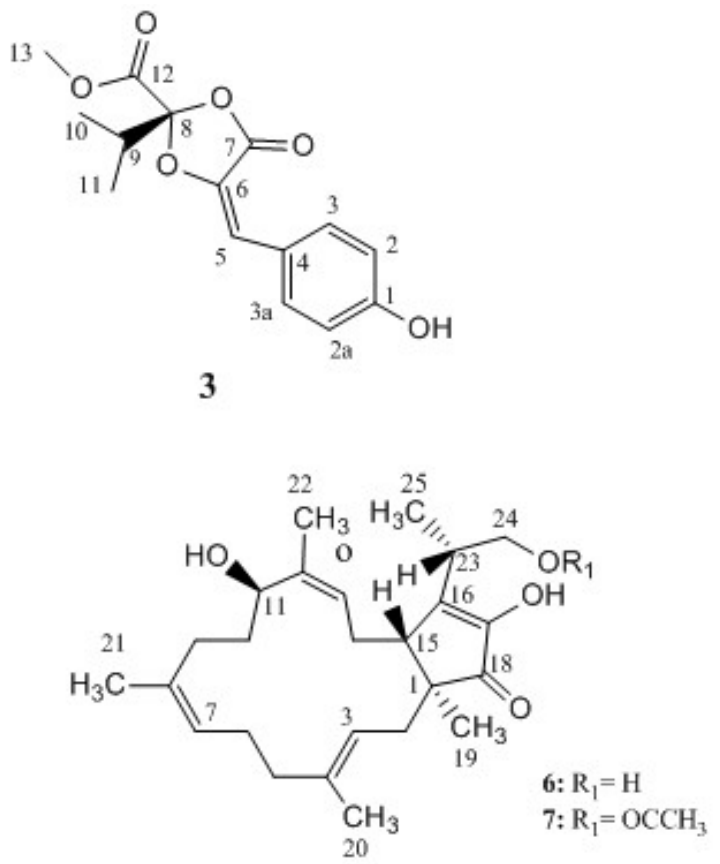

Fig.11 The chemical structure of compounds 1-7 


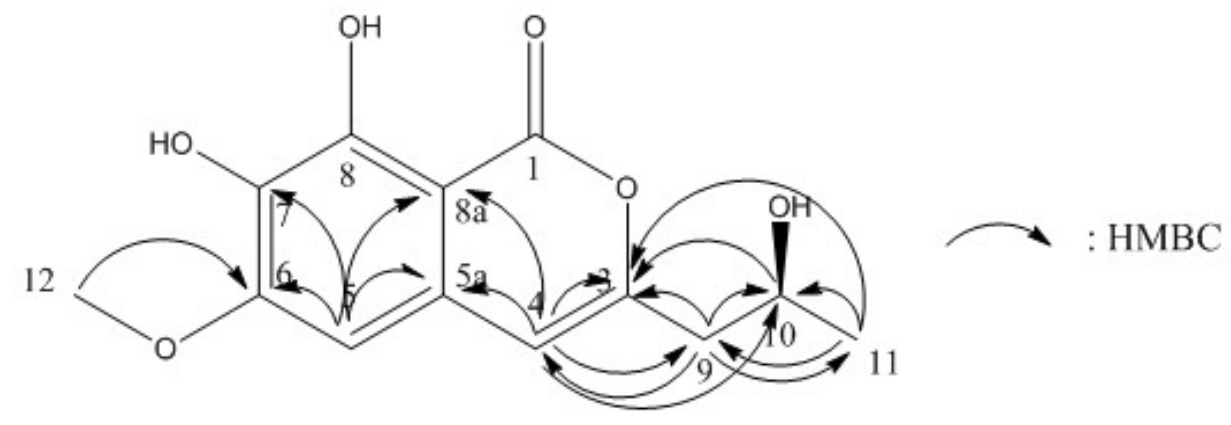

Fig.12. HMBC correlations of compound 1

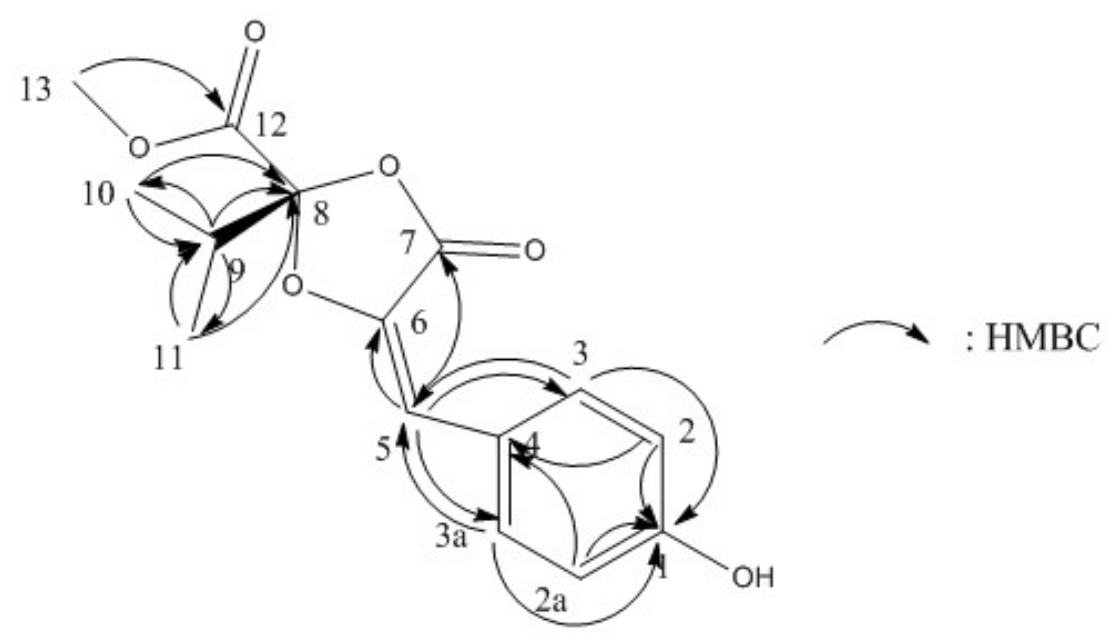

Fig.13 HMBC correlations of compound 3 


\section{The CD spectra of compound 3}

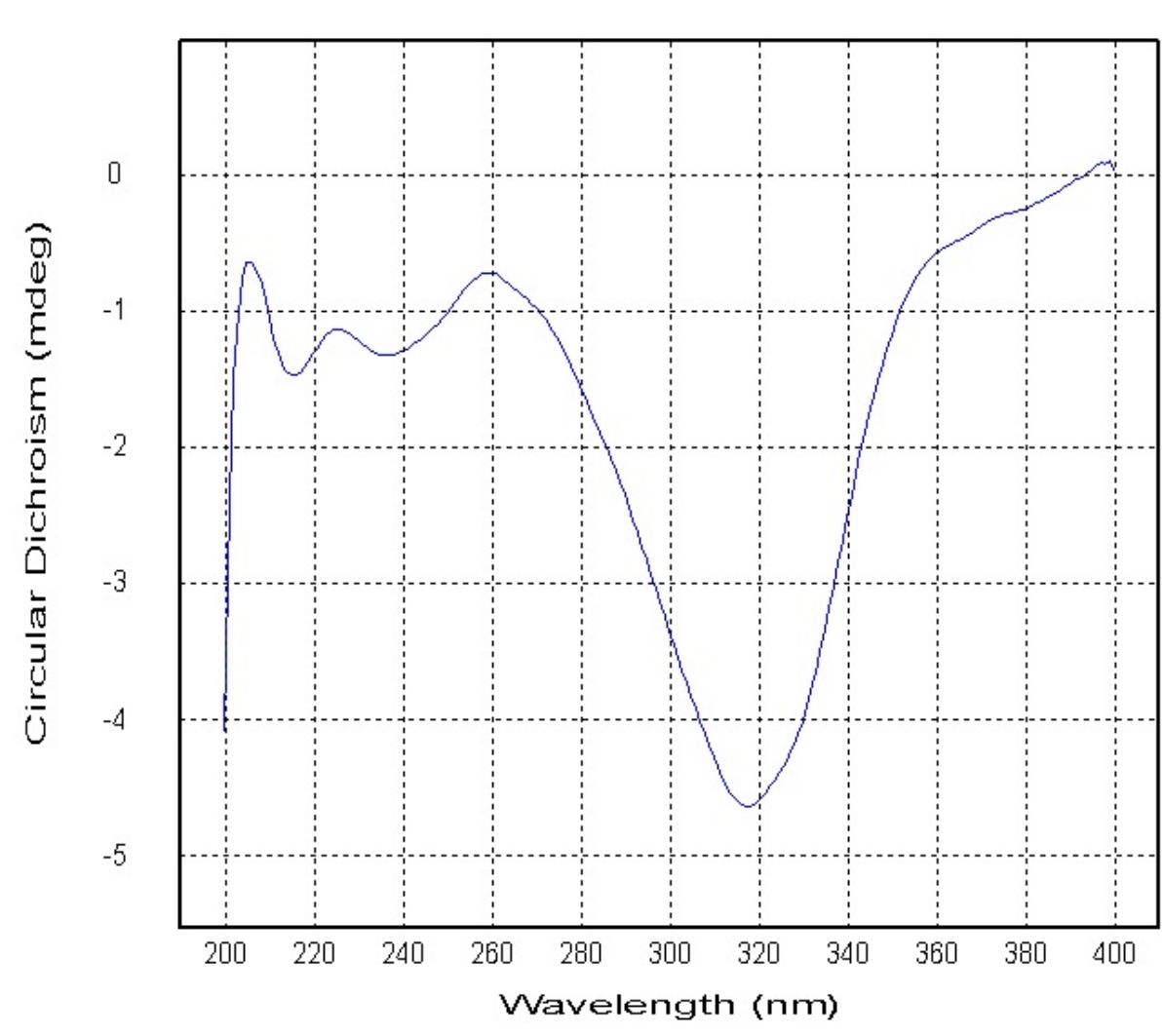

$\overline{\text { Smooth (s):1 }}$

Fig.14 The CD spectra of compound 3 


\section{Spectroscopic data of the isolated compounds}

Compound 2: Needles, $[\alpha]_{\mathrm{D}}^{25}+67.1\left(c\right.$ 1.0, $\left.\mathrm{CH}_{3} \mathrm{OH}\right)$, IR (KBr) $v_{\max }: 3333,1680,694 \mathrm{~cm}^{-1} ;{ }^{1} \mathrm{H}-\mathrm{NMR}(500 \mathrm{MHz}, \mathrm{MeOD}), \delta: 6.34(1 \mathrm{H}, \mathrm{s}, \mathrm{H}-4), 6.27$ (1H, s, H-7), 6.26 (1H, s, H-5), 4.15 (1H, q, H-10), 2.58 (2H, m, H-9), 1.25 (3H, d, $J=6.2 \mathrm{~Hz}, \mathrm{H}-11)$ ) ${ }^{13} \mathrm{C}-\mathrm{NMR}(125 \mathrm{MHz}, \mathrm{MeOD}), \delta: 23.3$ (C-11), 43.8 (C-9), 66.2 (C-10), 99.0 (C-8a), 102.9 (C-7), 104.4 (C-5), 107.1 (C-4), 141.1 (C-5a), 155.8 (C-3), 164.8 (C-8), 167.9 (C-6), 169.1 (C-1). HREIMS at $\mathrm{m} / z$ 259.0573 [M+Na] ${ }^{+}$(calcd for $\mathrm{C}_{12} \mathrm{H}_{12} \mathrm{NaO}_{5}$ ).

Compound 4 : Brown oil; ${ }^{1} \mathrm{H}$-NMR (500 MHz, MeOD), $\delta: 1.85\left(1 \mathrm{H}, \mathrm{ddd}, J=12.5,10.4,9.1 \mathrm{~Hz}, \mathrm{CH}_{2}(3)\right), 2.36\left(1 \mathrm{H}, \mathrm{ddd}, J=12.5,4.9,3.2 \mathrm{~Hz}, \mathrm{CH}_{2}(3)\right), 3.94$ $(1 \mathrm{H}, \mathrm{ddd}, J=10.3,6.8,3.2 \mathrm{~Hz}, \mathrm{H}-2), 4.78(1 \mathrm{H}, \mathrm{dd}, J=9.0,5.0 \mathrm{~Hz}, \mathrm{H}-1), 4.83(1 \mathrm{H}, \mathrm{d}, J=6.8 \mathrm{~Hz}, \mathrm{H}-4), 6.74(1 \mathrm{H}, \mathrm{d}, J=7.9 \mathrm{~Hz}, \mathrm{H}-8), 7.02(1 \mathrm{H}, \mathrm{d}, J=7.7 \mathrm{~Hz}, \mathrm{H}-6)$, $7.17(1 \mathrm{H}, \mathrm{t}, J=7.9 \mathrm{~Hz}, \mathrm{H}-7) ;{ }^{13} \mathrm{C}-\mathrm{NMR}$ (125MHz, MeOD), $\delta: 39.2$ (C-3), 68.6 (C-4), 72.2 (C-2), 73.7 (C-1), 115.7 (C-8), 119.3 (C-6), 123.0 (C-5a), 129.9 (C-7), 141.9 (C-8a), 157.9 (C-5).

Compound 5 : Brown oil; ${ }^{1} \mathrm{H}$-NMR (500 MHz, MeOD), $\delta: 1.93\left(1 \mathrm{H}, \mathrm{m}, \mathrm{CH}_{2}(3)\right), 2.14\left(1 \mathrm{H}, \mathrm{m}, \mathrm{CH}_{2}(3)\right), 4.19(1 \mathrm{H}, \mathrm{ddd}, J=9.1,6.0,3.1 \mathrm{~Hz}, \mathrm{H}-2), 4.73(1 \mathrm{H}, \mathrm{d}$, $J=6.0 \mathrm{~Hz}, \mathrm{H}-1), 4.82(1 \mathrm{H}, \mathrm{m}, \mathrm{H}-4), 6.73(1 \mathrm{H}, \mathrm{m}, \mathrm{H}-8), 6.93(1 \mathrm{H}, \mathrm{d}, J=7.6 \mathrm{~Hz}, \mathrm{H}-6), 7.13$ (1H, t, $J=7.8 \mathrm{~Hz}, \mathrm{H}-7) ;{ }^{13} \mathrm{C}-\mathrm{NMR}(125 \mathrm{MHz}, \mathrm{MeOD}), \delta: 37.4(\mathrm{C}-3), 67.7$ (C-4), 70.3 (C-2), 72.2 (C-1), 115.7 (C-8), 120.5 (C-6), 123.7 (C-5a), 129.8 (C-7), 140.9 (C-8a), 158.1(C-5).

Compound 6 : Yellow oil; ${ }^{1} \mathrm{H}$-NMR $\left(500 \mathrm{MHz}, \mathrm{CDCl}_{3}\right), \delta: 0.88$ (3H, s, H-19), 1.19 (3H, d, J=6.5 Hz, H-25), 1.48 (3H, s, H-22), 1.54 (6H, s, H-20, H-21), $1.66(2 \mathrm{H}, \mathrm{dd}, J=27.1,9.9 \mathrm{~Hz}, \mathrm{H}-2), 1.83(1 \mathrm{H}, \mathrm{m}, \mathrm{H}-10), 1.91(1 \mathrm{H}, \mathrm{t}, J=11.3 \mathrm{~Hz}, \mathrm{H}-14), 1.99$ (2H, d, $J=10.7 \mathrm{~Hz}, \mathrm{H}-9), 2.17$ (2H, d, $J=10.3 \mathrm{~Hz}, \mathrm{H}-5$,), $2.25(1 \mathrm{H}$, m, H-5,), 2.26 (1H, t, $J=11.9 \mathrm{~Hz}, \mathrm{H}-14), 2.34$ (1H, d, $J=16.5 \mathrm{~Hz}, \mathrm{H}-10), 2.59$ (2H, dd, $J=22.1,8.5 \mathrm{~Hz}, \mathrm{H}-6), 3.76$ (2H, dd, $J=26.2,19.5 \mathrm{~Hz}, \mathrm{H}-24), 3.96(1 \mathrm{H}, \mathrm{d}, J$ $=9.0 \mathrm{~Hz}, \mathrm{H}-11), 5.13(1 \mathrm{H}, \mathrm{s}, \mathrm{H}-7), 5.25(1 \mathrm{H}, \mathrm{s}, \mathrm{H}-3), 5.38$ (1H, m, H-13); ${ }^{13} \mathrm{C}-\mathrm{NMR}\left(125 \mathrm{MHz}, \mathrm{CDCl}_{3}\right), \delta: 10.3(\mathrm{C}-22), 14.4$ (C-25), $15.2(\mathrm{C}-21), 15.5(\mathrm{C}-20), 16.3$ (C-19), 23.8 (C-6), 28.8 (C-14), 29.7 (C-10), 34.9 (C-9), 37.12 (C-23), 39.3 (C-2), 40.3 (C-5), 49.1 (C-1), 49.4 (C-15), 65.6 (C-24), 76.4 (C-11), 121.7 (C-3), 124.3 (C-7), 129.2 (C-13), 132.7 (C-8), 136.1 (C-4), 137.8 (C-12), 147.5 (C-16), 151.1 (C-17), 209.1 (C-18).

Compound 7 : Yellow oil; ${ }^{1} \mathrm{H}-\mathrm{NMR}(500 \mathrm{MHz}, \mathrm{DMSO}), \delta: 0.88(3 \mathrm{H}, \mathrm{s}, \mathrm{H}-19), 1.22(3 \mathrm{H}, \mathrm{t}, J=8.5 \mathrm{~Hz}, \mathrm{H}-25), 1.48(3 \mathrm{H}, \mathrm{d}, J=13.2 \mathrm{~Hz}, \mathrm{H}-22), 1.59(6 \mathrm{H}, \mathrm{s}, \mathrm{H}-20$, $\mathrm{H}-21), 1.70(1 \mathrm{H}, \mathrm{t}, J=13.0 \mathrm{~Hz}, \mathrm{H}-10), 1.84(1 \mathrm{H}, \mathrm{m}, \mathrm{H}-10), 1.92(1 \mathrm{H}, \mathrm{m}, \mathrm{H}-14), 2.00\left(3 \mathrm{H}, \mathrm{s}, \mathrm{COCH}_{3}\right), 2.05(1 \mathrm{H}, \mathrm{d}, J=13.7 \mathrm{~Hz}, \mathrm{H}-5),, 2.25(1 \mathrm{H}, \mathrm{m}, \mathrm{H}-5),, 2.26(2 \mathrm{H}, \mathrm{m}$, H-6), $2.51(1 \mathrm{H}, \mathrm{s}, \mathrm{H}-14), 2.62(1 \mathrm{H}, \mathrm{d}, J=11.2 \mathrm{~Hz}, \mathrm{H}-23), 2.72(1 \mathrm{H}, \mathrm{m}, \mathrm{H}-15), 4.18(2 \mathrm{H}, \mathrm{d}, J=7.6 \mathrm{~Hz}, \mathrm{H}-24), 4.44$ (1H, t, $J=7.8 \mathrm{~Hz}, \mathrm{H}-11), 5.11(1 \mathrm{H}, \mathrm{s}, \mathrm{H}-7), 5.23$ (1H, s, H-13), 5.28 (1H, m, H-3); ${ }^{13} \mathrm{C}-\mathrm{NMR}$ (125MHz, DMSO), $\delta: 10.3$ (C-22), 14.2 (C-25), 15.0 (C-20), 15.7 (C-21), $15.9(\mathrm{C}-19), 20.7(\mathrm{COCH}), 23.3(\mathrm{C}-6), 28.5$ 
(C-14), 30.3 (C-10), 33.3(C-23), 35.3 (C-9), 39.6 (C-2), 40.7 (C-5), 48.3 (C-1), 48.5 (C-15), 65.7 (C-24), 74.2 (C-11), 121.9 (C-3), 123.3 (C-7), 126.8 (C-13), 132.5

(C-8), 136.8 (C-4), 136.9 (C-12), 147.8 (C-17), 147.8 (C-16), $170.2\left(\mathrm{COCH}_{3}\right), 207.3(\mathrm{C}-18)$.

\section{Table. ${ }^{1} \mathrm{H}$ and ${ }^{13} \mathrm{C}$-NMR data for $1-3$}

Table 1. ${ }^{1} \mathrm{H}$ and ${ }^{13} \mathrm{C}-\mathrm{NMR}$ data $(500 / 125 \mathrm{MHz})$ for $\mathbf{1}$ and 2 in MeOD

\begin{tabular}{|c|c|c|c|c|}
\hline \multirow{2}{*}{ No. } & \multicolumn{2}{|l|}{1} & \multicolumn{2}{|c|}{2} \\
\hline & $\delta_{\mathrm{H}}(J$ in $\mathrm{Hz})$ & $\delta_{\mathrm{C}}$ & $\delta_{\mathrm{H}} \quad(J$ in $\mathrm{Hz})$ & $\delta_{\mathrm{C}}$ \\
\hline 1 & & 167.9 & & 169.1 \\
\hline 3 & & 156.0 & & 155.8 \\
\hline 4 & $6.61 \mathrm{~s}$ & 101.7 & $6.34 \mathrm{~s}$ & 107.1 \\
\hline 5 & $6.38 \mathrm{~s}$ & 103.9 & $6.26 \mathrm{~s}$ & 104.4 \\
\hline $5 a$ & & 132.4 & & 141.1 \\
\hline 6 & & 161.0 & & 167.9 \\
\hline 7 & & 136.0 & $6.27 \mathrm{~s}$ & 102.9 \\
\hline 8 & & 161.7 & & 164.8 \\
\hline $8 \mathrm{a}$ & & 98.3 & & 99.0 \\
\hline 9 & $2.62 \mathrm{~m}$ & 44.2 & $2.58 \mathrm{~m}$ & 43.8 \\
\hline 10 & $4.16 \mathrm{~m}$ & 66.4 & $4.15 \mathrm{q}$ & 66.2 \\
\hline 12 & $3.76 \mathrm{~s}$ & 61.8 & & \\
\hline
\end{tabular}


Table $2 .{ }^{1} \mathrm{H},{ }^{13} \mathrm{C}-\mathrm{NMR}(500 / 125 \mathrm{MHz})$ and $\mathrm{HMBC}$ data for compound 3 in $\mathrm{CDCl}_{3}$

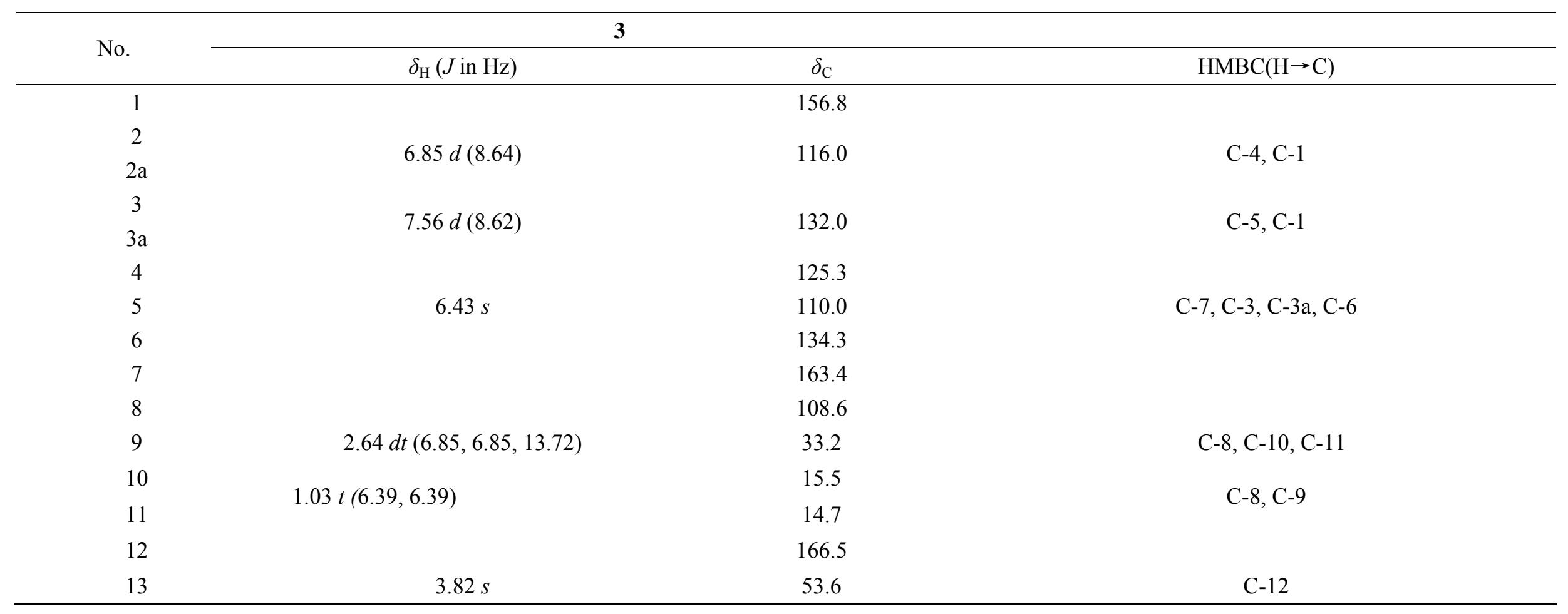


6. COX-2 inhibitory value of Compound 3

\begin{tabular}{|l|c|c|c|c|}
\hline & WJF-53 & WJF-55 & Compound 3 & BD11 \\
\hline IC50 & $1.052 \mathrm{E}-05$ & $1.92 \mathrm{E}-06$ & $1.118 \mathrm{E}-05$ & $8.483 \mathrm{E}-06$ \\
\hline
\end{tabular}

Graphics of COX-2 inhibitory value of Compound 3

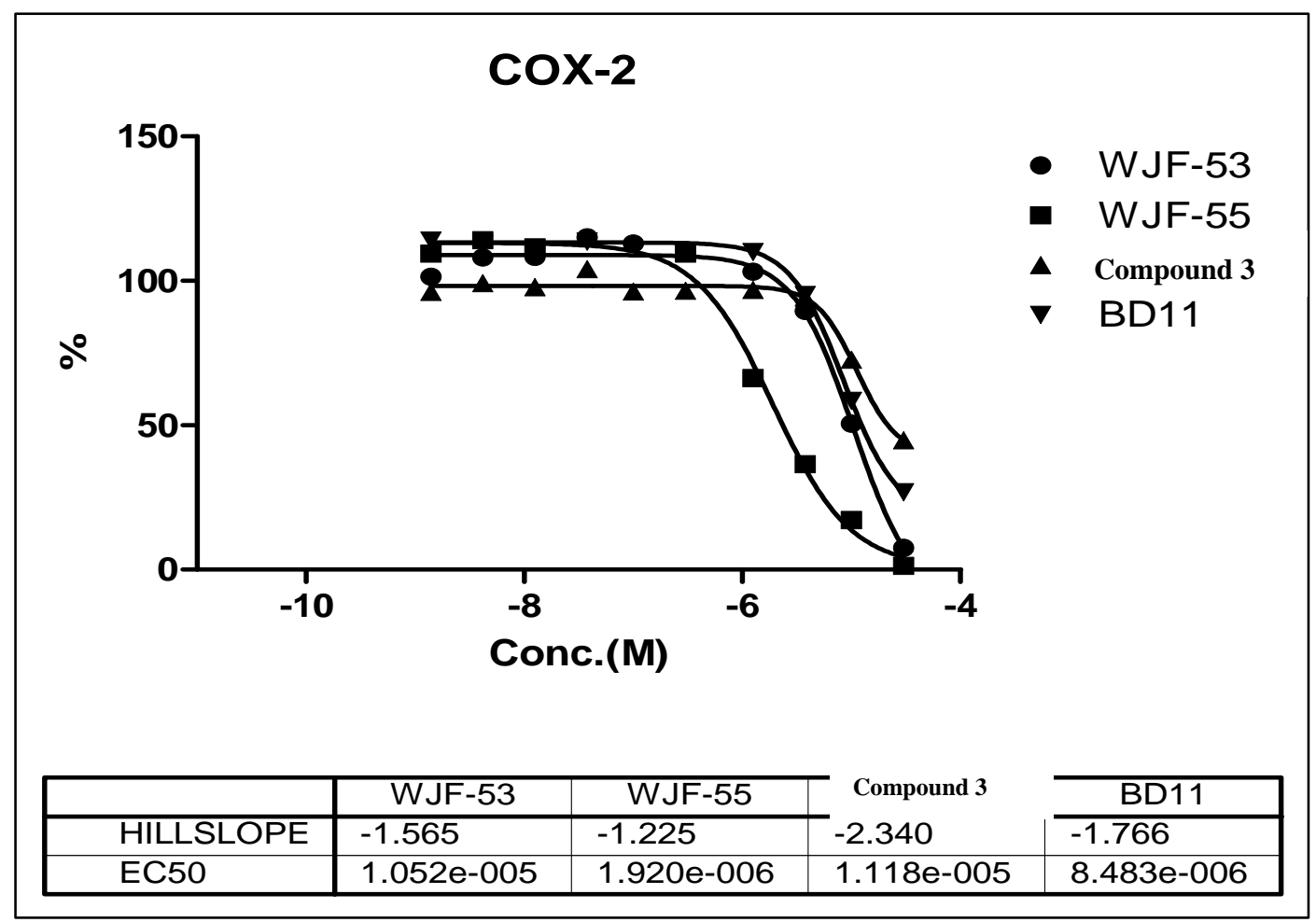

